\title{
LAMINAR FREE-SURFACE FLOW INTO A VERTICAL CYLINDER
}

\author{
THOMAS G. SMITH \\ Department of Chemical Engineering, University of Notre Dame, \\ Notre Dame, Indiana, 46556, U.S.A. \\ and \\ J. O. WILKES \\ Department of Chemical Engineering, The University of Michigan, \\ Ann Arbor, Michigan, U.S.A.
}

(Received 10 August 1973)

\begin{abstract}
This study concerns the unsteady free-surface flow of a Newtonian fluid issuing vertically upwards from a tube in the base of a vertical cylindrical cavity. Experimental and numerical results are presented and discussed.

The experiments involve a photographic procedure for measuring point velocities and surface profiles in an unsteady velocity field. Numerically, a marker-and-cell technique is used in conjunction with a Dufort-Frankel finite-difference approximation for solving the Navier-Stokes equation in two-dimensional cylindrical coordinates. It is found that the numerical solutions are generally stable for large values of the time step in spite of high fluid viscosities. Good agreement is obtained between the numerical and experimental results.
\end{abstract}

\section{INTRODUCTION}

THE WORK reported is concerned with the numerical solution of the nonlinear equations of motion describing the unsteady, highly viscous, free-surface flow of a Newtonian fluid issuing vertically, with mean velocity $\bar{V}$, from a cylindrical tube into the bottom of a vertical cylindrical enclosure (Fig. 1). Conditions are isothermal and gravitational effects are taken into account.

The fluid in the inlet tube is initially motionless. Subsequently, it flows into the bottom of the cavity in either a non-jet or jet-like manner, depending on its velocity, eventually impinging on the walls of the enclosure and advancing upward. The investigation can be viewed as a prelude to a more systematic study of such topics as injection molding, viscous unsteady and steady-state jets, and surface diffusion phenomena.

Two particular difficulties of the stated problem govern the direction to be taken in selecting an efficient numerical approach. First, the presence of a high viscosity liquid dictates that, for stability, a prohibitively small value of the time step may have to be used. To alleviate this limitation, the approach of Dufort and Frankel[5] is employed. Instabilities having their origin in the inertial terms may also occur, and in this respect the results of Williams[23] are of special interest.

The second difficulty concerns the moving free surface. An effective technique for dealing with problems of this nature is the marker-and-cell (MAC) method developed by Welch et al.[22]. Although on initial examination the MAC scheme appears to be basically an elaborate and detailed sequence of book-keeping steps, it in fact leads to a significant change in the fundamental development of the finite-difference equations. In brief, the use of marker particles depends on the definition of cells. This in turn requires the use of 


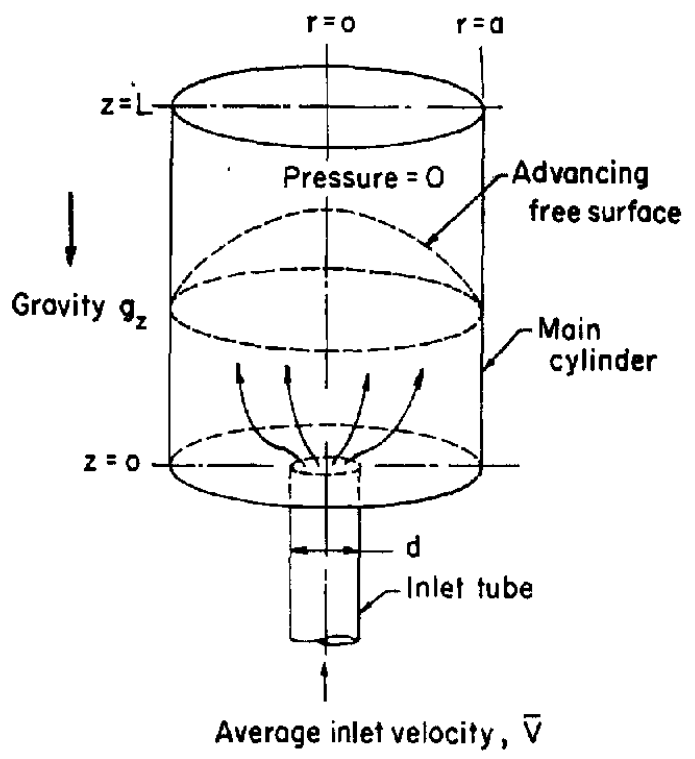

Fig. 1. Liquid injection into a vertical cylinder.

an interlaced grid system. Additionally, the retention of the Navier-Stokes equation in its basic velocity/pressure (primitive) form is convenient when working in two-dimensional cylindrical coordinates, three dimensions, or when free surfaces are involved. However, a way of satisfying continuity is now needed, and this is conveniently provided by defining a divergence or discrepancy term for each cell and using it to formulate an equation for the pressure.

Various free surface boundary conditions were tested and the simplest procedure that yielded reasonable agreement with experiment was used. The question of fluid motion in the vicinity of the contact line (see, e.g., Huh and Scriven[11]) was avoided in the numerical work by imagining the flow to occur into a vacuum.

The experimental program established to provide data suitable for verifying the numerical results is an extension of the work of Andrade and Tsien[1] and Atkinson et al.[2]. The experiments consist of measuring both velocities within the fluid and free-surface profiles. Computer simulations of the experimental runs are compared directly with experimental results.

\section{THE MATHEMATICAL MODEL}

The partial differential equations and boundary and initial conditions are developed for the physical situation illustrated in Fig. 1. A viscous Newtonian liquid of kinematic viscosity $v$ is injected into the bottom of an empty vertical cylindrical cavity of diameter $2 a$ through an inlet tube of smaller diameter $d$; the velocity distribution in the inlet tube is taken to be parabolic. The flow in the main cylindrical tube is considered to be unsteady, laminar, isothermal, and axially symmetric and involves a moving free surface. Nonlinear terms and gravitational acceleration, $g$, are included in the analysis. The objective is to compute the fluid velocities and the shape of the free surface as a function of time.

The complete equations governing this flow can be written as

$$
\nabla \cdot \mathbf{w}=\mathbf{0},
$$




$$
\frac{\partial \mathbf{w}}{\partial t}=-\nabla \cdot(\mathbf{w w})-\nabla \phi+v \nabla \cdot(\nabla \mathbf{w})+\mathbf{g}
$$

Here, $w$ is the velocity vector, $t$ is time, $\phi$ is pressure divided by fluid density $\rho$, and $g$ is the gravitational vector. These equations represent mass and momentum balances for a volume fixed in space and are the appropriate forms for the numerical technique used in their solution. In particular, the form of the convection terms as $\nabla \cdot(w w)$ rather than $(w \cdot \nabla) w$ is important for the stability of the numerical method, as will be discussed in $\S 3$. The form of the viscous term is required for compatibility with the Dufort-Frankel finite-difference approximation (FDA) and consequently should not be simplified by means of the incompressibility condition.

With $u$ and $v$ the velocity components in the $r$ and $z$ directions, respectively, (1) and (2) can be written in two-dimensional cylindrical coordinates as

$$
\begin{gathered}
D \equiv \frac{1}{r} \frac{\partial(r u)}{\partial r}+\frac{\partial v}{\partial z}=0 \\
\frac{\partial u}{\partial t}+\frac{1}{r} \frac{\partial\left(r u^{2}\right)}{\partial r}+\frac{\partial(u v)}{\partial z}=-\frac{\partial \phi}{\partial r}+v\left(\frac{\partial^{2} u}{\partial r^{2}}+\frac{1}{r} \frac{\partial u}{\partial r}-\frac{u}{r^{2}}+\frac{\partial^{2} u}{\partial z^{2}}\right), \\
\frac{\partial v}{\partial t}+\frac{1}{r} \frac{\partial(r u v)}{\partial r}+\frac{\partial\left(v^{2}\right)}{\partial z}=-\frac{\partial \phi}{\partial z}+v\left(\frac{\partial^{2} v}{\partial r^{2}}+\frac{1}{r} \frac{\partial v}{\partial r}+\frac{\partial^{2} v}{\partial z^{2}}\right)-g_{z} .
\end{gathered}
$$

An equation for the pressure at any time is obtained by differentiating (3), (4), and (5) with respect to $t, r$, and $z$, respectively, and eliminating time derivatives of $u$ and $v$, giving

$$
\frac{\partial^{2} \phi}{\partial r^{2}}+\frac{1}{r} \frac{\partial \phi}{\partial r}+\frac{\partial^{2} \phi}{\partial z^{2}}+I=-\frac{\partial D}{\partial t}+v \nabla^{2} D
$$

where

$$
I=\frac{1}{r} \frac{\partial^{2}\left(r u^{2}\right)}{\partial r^{2}}+\frac{2}{r} \frac{\partial^{2}(r u v)}{\partial r \partial z}+\frac{\partial^{2}\left(v^{2}\right)}{\partial z^{2}}
$$

Although the divergence $D$ is identically zero in the continuous problem, its finite-difference counterpart will not necessarily be zero, in which case corrective action must be taken by suitable modification of the pressure field. The indicated derivatives of $D$ provide the appropriate adjustment. Thus, (6) can be viewed as a predictive equation for the incompressibility condition.

Boundary conditions at the solid surfaces, free surface, and inlet must be prescribed for (4), (5), and (6), together with initial conditions for (4) and (5). Initially, the cylindrical cavity is empty and the fluid in the inlet tube is at rest. Subsequently, all velocities at solid boundaries remain zero. Symmetry of velocity relative to the centerline is assumed and in the inlet region the velocity distribution is taken to be parabolic. The solid surface and inlet boundary conditions for (6) are obtained from (4) and (5) which relate the normal pressure derivatives to the body and viscous forces. The implementation of these concepts in terms of a finite-difference methodology is summarized briefly in $\$ 3$, as are the free-surface boundary conditions.

Several assumptions made in developing the above model warrant review and justification. The assumptions fall into two categories, namely, those that can be precisely satisfied by a 
suitable design of the experiments and those that, without a great deal of difficulty, can not. The latter are dealt with in the remainder of this section.

It has been assumed that a parabolic velocity profile exists at $z=0$ and $t=0$. There are at least six factors to consider in justifying this assumption. First, the time required to accelerate the fluid in the inlet tube from rest to a steady-state velocity profile must be calculated. Using the analysis of Szymanski, as presented in Schlichting[18], the acceleration time is typically less than 10 per cent of the total duration of a given experiment. However, for those experiments involving the lower viscosity fluids, the acceleration time becomes relatively quite large, and at high inlet velocities this effect may then exert a noticeable influence on the overall fluid mechanics in the main cylindrical tube. Second, at low Reynolds numbers, the interaction of the flow fields upstream and downstream of $z=0$ is thought to cause a departure from parabolic flow within the inlet tube near $z=0$ (see, e.g., Richardson[17]; Tillett[21]. The details of this disturbance are presently not well understood but it is known experimentally (Goren and Wronski[8]) that an increase in jet diameter can occur. In the present problem, gravity tends to increase the diameter and is undoubtedly the dominant force in this respect. Therefore, the use of a parabolic velocity profile at $z=0$ seems reasonable. The other four effects relating to the parabolic assumption are: the pressure drop history during the time interval of the experiment, the length of inlet tube required to achieve fully developed flow from an entering non-parabolic profile, the length of inlet tube required to dampen out any swirling velocity components, and viscous heating effects. Their influence is noted in $\$ 4$.

It is readily found that the flow and pressure fields are governed by the Reynolds number, $\operatorname{Re}=d \bar{V} / v$, the Froude number, $\mathrm{Fr}=\bar{V}^{2} / \mathrm{d} g_{z}$, and by the diameter ratio $d / 2 a$. Since the solution is a function of two dimensionless groups, $\mathrm{Re}$ and $\mathrm{Fr}$, having certain parameters in common but to a different power, there is obviously no gain in generality in using dimensionless equations.

\section{THE NUMERICAL PROCEDURE}

\subsection{System of grid points}

The basic cell concept dictates the construction of the grid point network. For a given cell, the field variable and indexing arrangement is illustrated in Fig. 2. The cell concept

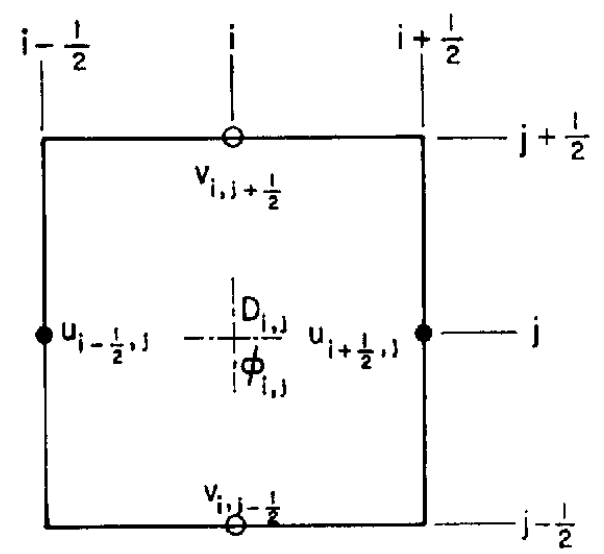

Fig. 2. Field-variable and indexing arrangement for cell $(i, j)$. 
was used by Welch et al.[22] and has been proposed as being necessary to insure mass and momentum conservation (Williams[23]).

Figure 3 represents the region of interest in terms of a rectangular mesh of these cells. The ranges of coordinates are $0 \leq r \leq a, 0 \leq z \leq L$ and the cell dimensions are

$$
r=a /(M-1), \quad z=L /(N-1),
$$

where $M$ and $N$ are integers. A cell $(\mathrm{i}, \mathrm{j})$ is such that its edges farthest from the origin have radial and axial coordinates

$$
r=(i-1) \Delta r, \quad z=(j-1) \Delta z,
$$

respectively. The indices $i$ and $j$ refer to the cell center. Cell boundaries are designated as $i+1 / 2, i-1 / 2, j+1 / 2$ and $j-1 / 2$. To facilitate the application of boundary conditions, the entire computational region is surrounded by either border cells or inlet cells as shown in Fig. 3.

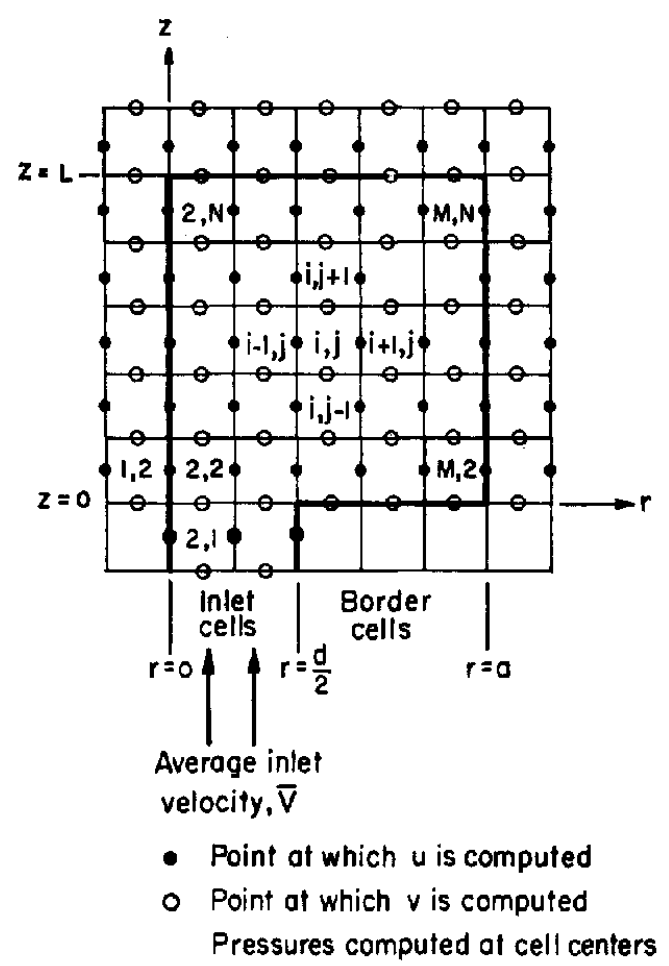

Fig. 3. Arrangement of cells for the finite-difference calculations.

\subsection{The finite-difference equations}

In the following FDA to (3), (4), and (5) the superscript locates the time level and, when omitted, $\mathrm{n}$ is implied:

$$
D_{i, j}=\frac{1}{r_{i} \Delta r}\left[(r u)_{i+1 / 2, j}-(r u)_{i-1 / 2, j}\right]+\frac{v_{i, j+1 / 2}-v_{i, j-1 / 2}}{\Delta z},
$$




$$
\begin{aligned}
\frac{u_{i+1 / 2, j}^{n+1}-u_{i+1 / 2, j}^{n-1}}{2 \Delta t} & =\frac{1}{r_{i+1 / 2} \Delta r}\left[\left(r u^{2}\right)_{i, j}-\left(r u^{2}\right)_{i+1, j}\right] \\
& +\frac{(u v)_{i+1 / 2, j-1 / 2}-(u v)_{i+1 / 2, j+1 / 2}}{\Delta z}+\frac{\phi_{i, j}-\phi_{i+1, j}}{\Delta r} \\
& +v\left[\frac{u_{i+3 / 2, j}+u_{i-1 / 2, j}-u_{i+1 / 2, j}^{n+1}-u_{i+1 / 2, j}^{n-1}}{(\Delta r)^{2}}\right. \\
& +\frac{1}{r_{i+1 / 2}}\left(\frac{u_{i+1, j}-u_{i, j}}{\Delta r}\right)-\frac{u_{i+1 / 2, j}^{n+1}+u_{i+1 / 2, j}^{n-1}}{2 r_{i+1 / 2}^{2}} \\
& \left.+\frac{u_{i+1 / 2, j+1}+u_{i+1 / 2, j-1}-u_{i+1 / 2, j}^{n+1}-u_{i+1 / 2, j}^{n-1}}{(\Delta z)^{2}}\right] \\
\frac{v_{i, j+1 / 2}^{n+1}-v_{i, j+1 / 2}^{n-1}=}{2 \Delta t} & \frac{1}{r_{i} \Delta r}\left[(r u v)_{i-1 / 2, j+1 / 2}-(r u v)_{i+1 / 2, j+1 / 2}\right] \\
& \left.+\frac{v_{i, j}^{2}-v_{i, j+1}^{2}+\frac{\phi_{i, j}-\phi_{i, j+1}}{\Delta z}-g_{z}}{\Delta z}\right] \\
& +v\left[\frac{v_{i, j+3 / 2}+v_{i, j-1 / 2}-v_{i, j+1 / 2}^{n+1}-v_{i, j+1 / 2}^{n-1}}{(\Delta z)^{2}}\right. \\
& +\frac{v_{i+1 / 2, j+1 / 2}-v_{i-1 / 2, j+1 / 2}}{r_{i} \Delta r} \\
& +\frac{\left.v_{i+1, j+1 / 2}+v_{i-1, j+1 / 2}-v_{i, j+1 / 2}^{n+1}-v_{i, j+1 / 2}^{n-1}\right]}{(\Delta r)^{2}}
\end{aligned}
$$

Truncation errors for these equations are of order $(\Delta r)^{2},(\Delta z)^{2}$, and $(\Delta t)^{2}$. Averaging of adjacent variables is obviously required for points where these variables are not explicitly computed. For example,

$$
(u v)_{i-1 / 2, j-1 / 2}=\left(\frac{u_{i-1 / 2, j}+u_{i-1 / 2, j-1}}{2}\right)\left(\frac{v_{i, j-1 / 2}+v_{i-1, j-1 / 2}}{2}\right) .
$$

If existing fields of $u, v$, and $\phi$ are known at time level $n$, then (9) and (10), when applied to each cell in turn, are immediately appropriate for the calculation of new velocities at time level $n+1$, provided that all cells involved are full of fluid. The computation of velocities in surface and border cells will be discussed in $\$ 3 \cdot 2$ and $\$ 3 \cdot 3$. After calculating the velocities at an advanced time level, the new pressure field corresponding to this velocity field is calculated. This is accomplished using the FDA to (6), rearranged as follows:

$$
\begin{aligned}
\phi_{i, j}= & \phi_{i, j}+\frac{\omega}{4}\left\{\left(1+\frac{\Delta r}{2 r_{i}}\right) \phi_{i+1, j}+\left(1-\frac{\Delta r}{2 r_{i}}\right) \phi_{i-1, j}+\phi_{i, j+1}+\phi_{i, j-1}-4 \phi_{i, j}-\frac{(\Delta r)^{2}}{\Delta t} D_{i, j}\right. \\
& -v\left[\left(1+\frac{\Delta r}{2 r_{i}}\right) D_{i+1, j}+\left(1-\frac{\Delta r}{2 r_{i}}\right) D_{i-1, j}+D_{i, j+1}+D_{i, j-1}-4 D_{i, j}\right] \\
& +\frac{1}{r_{i}}\left[\left(r u^{2}\right)_{i+1, j}+\left(r u^{2}\right)_{i-1, j}-2\left(r u^{2}\right)_{i, j}\right]+v_{i, j+1}^{2}+v_{i, j-1}^{2}-2 v_{i, j}^{2} \\
& \left.+\frac{2}{r_{i}}\left[(r u v)_{i+1 / 2, j+1 / 2}+(r u v)_{i-1 / 2, j-1 / 2}-(r u v)_{i+1 / 2, j-1 / 2}-(r u v)_{i-1 / 2, j+1 / 2}\right]\right\} .(11)
\end{aligned}
$$


Here $\omega$ is a relaxation parameter. The variables needed in (11), together with their location, are shown in Fig. 4 for a general cell $(i, j)$.

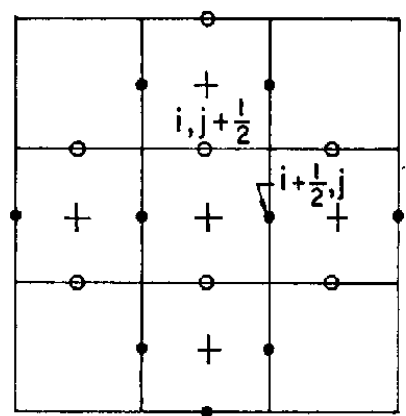

Fig. 4. Locations of the variables involved in the FDA of the presssure equation.

\subsection{Solution technique and stability.}

One of the main complications of the physical problem being considered is that it involves a free surface whose location must always be known. As mentioned previously, a computational device for dealing with free-surface problems in general has been developed and proven effective for several interesting cases (Harlow and Welch[9]). The technique as applied here is to simulate the introduction into the inlet stream of a number of initially regularly spaced massless marker particles, which are subsequently allowed to move at the local fluid velocity. The advancing free surface can then be located between pairs of cells, one of which contains particles and the other of which does not. These particles, whose motion describe the trajectories of the actual fluid, thus reveal to which cells, at any given time, the surface boundary conditions should be applied. This is the only manner in which the marker particles influence the computations. The procedure by which particles are created, identified, and moved, together with methods for the identification and relabelling of cells, are very detailed. The necessary steps for the problem at hand are too voluminous for presentation here and can be found in Smith[19].

One of the distinguishing features of the present work is the concern with high-viscosity fluids. In this respect, if the FDA to the continuous equations had been developed using a standard explicit formulation, the time step would have been restricted to $\Delta t \leq(\Delta x)^{2} / 4 v$ to suppress a strong numerical instability characteristically exhibited by the diffusion terms. For the present investigation, where viscosities as high as 60 poise are tested in the experimental program, the above stability criterion represents a serious computational limitation. An implicit alternating direction method has been considered but appears to present unnecessary computational complications when used in conjunction with the MAC approach. Instead, the Dufort-Frankel explicit technique, which is unconditionally stable for linear partial differential equations, is used in formulating (9) and (10) in the expectation that a less restrictive stability criterion will result for the nonlinear problem. On the other hand, since variables at three time levels are now involved, it is possible for a parasitic solution to develop, leading eventually to a weak instability known as time splitting (Platz$\operatorname{man}[16])$.

Note that use of the Dufort-Frankel method requires the retention of the friction terms in their complete form, $v \nabla \cdot(\nabla w)$ rather than their simplification, using incompressibility, to $v \nabla \times(\nabla \times \mathbf{w})$. 
The convective terms in (9) and (10) have been written in the total derivative form to control nonlinear computational instability. This, together with limiting the time-step increment to $\Delta t \leq \Delta z /\left|v_{\max }\right|$, insures stability (Williams[23]) because aliasing is controlled (see also Welch et al.[22]). Note that precise momentum conservation (Fox[6]) is prohibited in cylindrical coordinates.

In a rearranged form (see (6)), (11) is essentially a predictive equation for $\partial D / \partial t$, the rate of change of the divergence. One might expect that the desired rate of change of this quantity would be zero. However, a different approach is necessary. We first recognize the fact that the existing $D_{i, j}$ at time-level $n$ may be finite (i.e., continuity is not perfectly satisfied by the finite-difference solution), and force $D_{i, j}$ toward zero by proper adjustment of the new pressures. Therefore, $(\partial D / \partial t)_{i, j} \doteq\left(D_{i, j}^{n+1}-D_{i, j}\right) / \Delta t$ is taken as $-D_{i, j} / \Delta t$ in formulating (11). This procedure is required to prevent the growth of $D$ and consequent instability. Further, it has been shown that when (11) is solved iteratively, as it is here, the convergence criterion for the solution can be relaxed considerably by retaining the remaining artificial divergence terms, thus reducing computation time. To further minimize the time needed to solve (11), we have accelerated convergence by using the method of successive over-relaxation (Carnahan et al. [3]). The optimum value of the relaxation parameter, $\omega$, having a value between 1 and 2, can only be found heuristically for the present problem and in fact varies as the computational region changes, i.e., as the free surface advances. A value of 1.6 proved adequate for a majority of the cases, requiring between 5 and 50 iterations for a satisfactory degree of convergence.

\subsection{Boundary conditions}

The following strategy is employed in administering the free-surface boundary conditions. First, it is required that $D \equiv 0$ for surface cells. Satisfying continuity is especially important at the surface because of the absence of a pressure (as computed by (11)) in such cells to compensate for finite values of $D$. When a surface cell has only one free side (the most important case) it is possible to calculate three of the four surface cell velocities using (9) and (10). The fourth velocity, the free surface velocity, is then computed from (8) thus insuring $D \equiv 0$.

For most of the flows considered here, surface cells with two or more free sides occur much less frequently than those with one free side (this is accounted for by the fact that in the MAC method all surface cells are considered to be completely full even if they are only partially full) and, consequently, the particular procedure used for multiple free-side cells has a relatively minor effect on the overall velocity field.

The results presented in $\$ 5$ show that for the type of flow under consideration, the pressure in a free surface cell can be assigned the value of the external pressure, namely zero. The basis for this is that the relatively large tube diameter, approximately $1 \mathrm{~cm}$, reduces surface tension effects to a minor role, especially for higher viscosity fluids at reasonable velocities. Additionally, the results of calculations which included the influence of normal viscous stress on the surface cell pressure were not significantly different from those that neglected this effect.

At solid surfaces the boundary conditions are applied by means of the border cells shown in Fig. 3. The velocity and pressures in these cells are calculated using the boundary conditions stated in $\$ 2$ and by insisting that $D \equiv 0$. The full prediction equations for velocity and pressure can then be applied, without modification, near the boundaries. This avoids 
a complicated redefinition of these equations at and near the boundaries and is the reason for using border cells.

A complete development and listing of the finite-difference form of the boundary conditions is given by Smith[19]. Because of the many details involved, only the key guidelines are presented above.

\section{THE EXPERIMENT}

The equipment for the unsteady state experiments is depicted in Figs. 5 and 6. Certain elements of this equipment have been tested by means of preliminary experiments and proven satisfactory for measuring the fully established velocity distribution in Poiseuille flow. Steady-state velocities on the order of $5 \mathrm{~cm} / \mathrm{sec}$ can be determined to within 5 per cent. Unsteady free-surface flows present five additional difficulties: particle acceleration should be accounted for, velocities in two directions must be measured, flow calibration is more complicated, the free surface must be contended with, and a movie camera rather than a still camera is employed, leading to various difficulties.

The experimental method basically involves illuminating, from the side, particles having a high reflectance, viewing against a dark background, and photographing with a 16-mm movie camera. Subsequent measurements are made directly from the negatives.

Since the duration of the experiments is less than $10 \mathrm{sec}$, temperature control is unnecessary. Furthermore, viscous heating effects in the inlet tube are calculated to be negligible for the velocities, viscosities and diameters under consideration. Regarding this, Goren and Wronski[8] conclude that a point temperature rise of up to $5^{\circ} \mathrm{C}$ is allowable before the viscosity variation significantly affects the velocity distribution (see also Gerrard et al.[7]).

Dimethylpolysiloxane liquids having nominal viscosities of 1,10 , and 60 stokes are employed. Viscosities at various shear rates up to 200 reciprocal seconds are measured

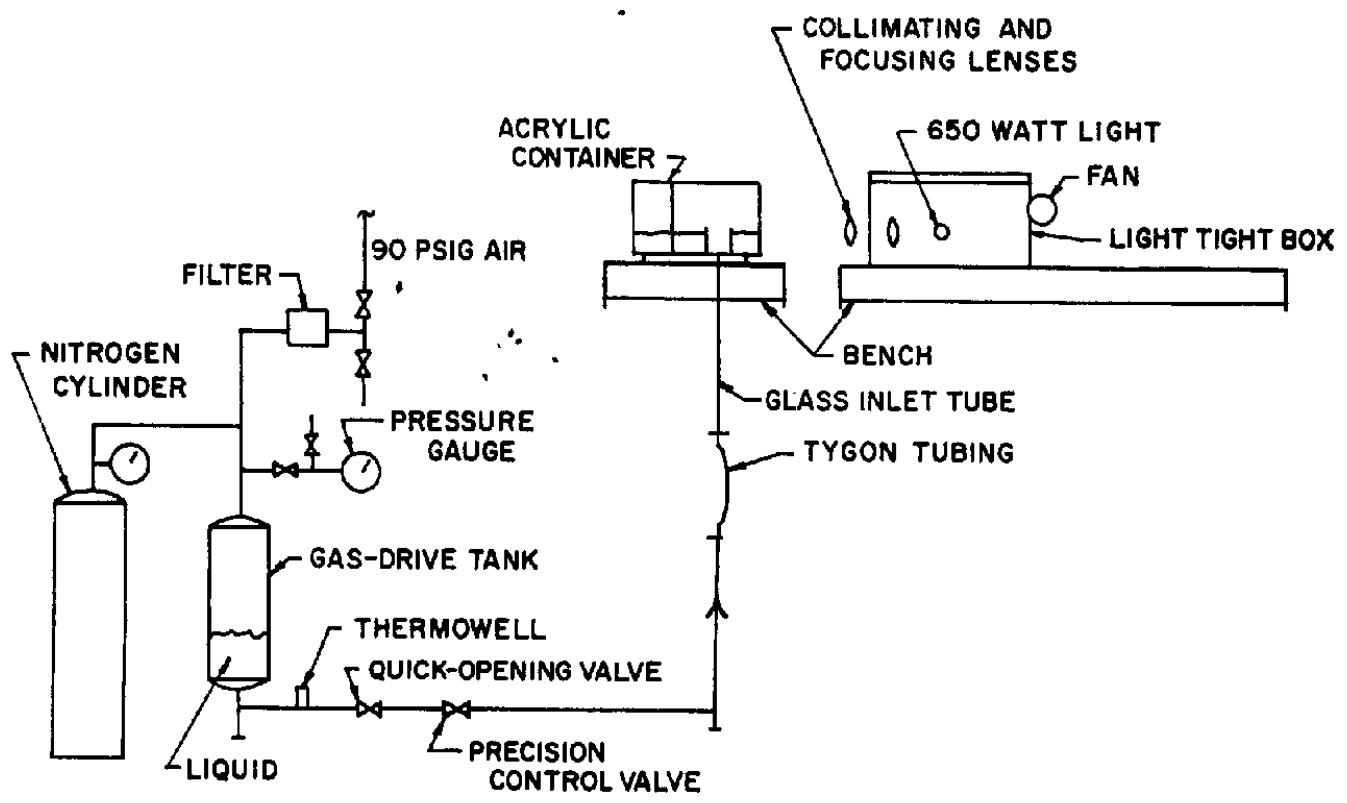

Fig. 5. Equipment flow diagram for the unsteady-state experiments. 


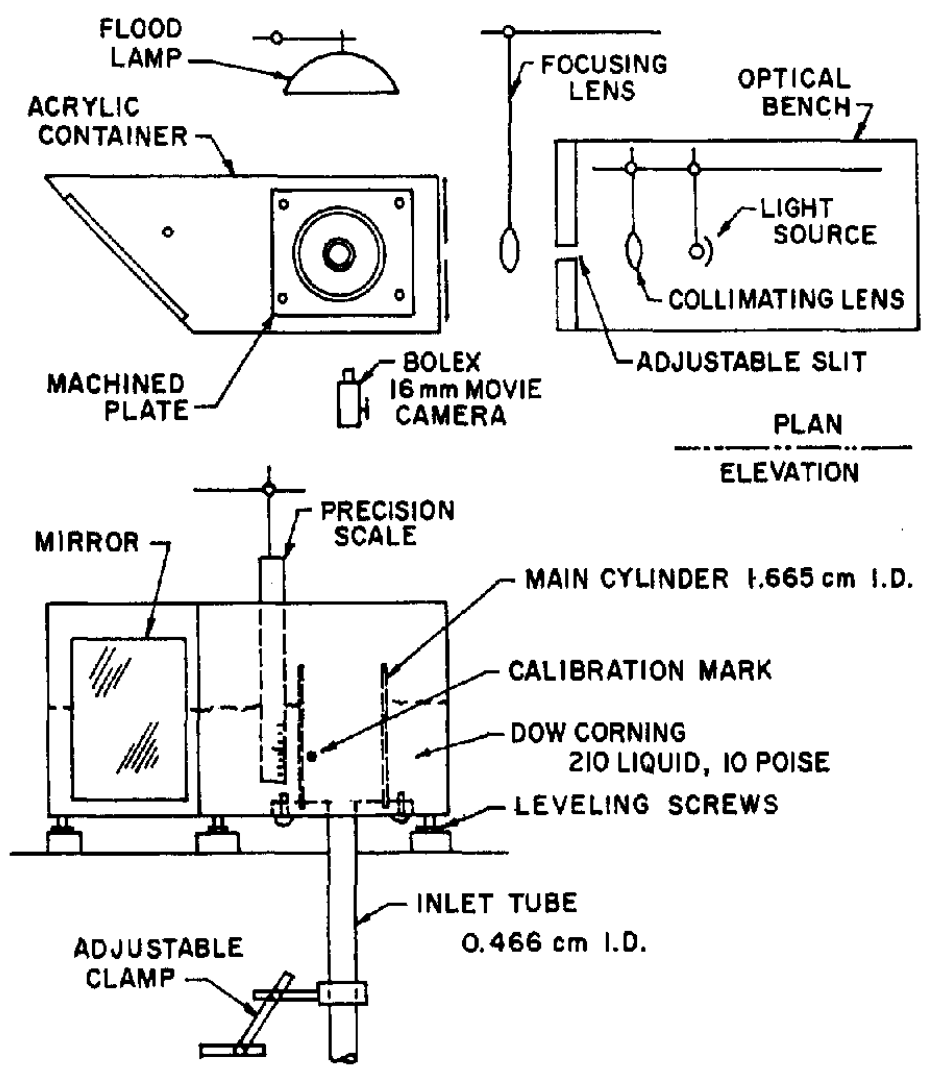

Fig. 6. Optical system.

with a Weissenberg rheogoniometer using the most viscous liquid. The behavior is Newtonian for this range of shear rates.

During a given experiment, liquid is forced from a reservoir into an open-ended glass cylindrical tube having an inner diameter of $2.565 \mathrm{~cm}$. Due to the large volume ratio of the reservoir to the cylindrical cavity, the pressure-drop history during a run is constant. Therefore, once a fully developed velocity profile has been established, the flow rate will remain constant. The lower end of the open-ended tube fits into a machined acrylic base plate. Precision-bore glass inlet tubes of inner diameter 0.466 and $1.665 \mathrm{~cm}$ are located concentrically in an appropriate base. The vertical alignment of the inlet tube and main tube is verified prior to each experiment. The inlet tube is $24 \mathrm{~cm}$ long, sufficient to insure a fully developed velocity profile (cf. Schlichting[18]) together with the damping out of any swirl velocity components (cf. Talbot[20]). No swirling is detected during the experiments.

Glass spheres in the range of 200-300 mesh serve as the tracer particles. A high-reflectance nacreous pigment in the form of extremely thin platelets has also been tried as a tracer, but is not successful unless used in a low viscosity liquid such as water. The difficulty is that the shear forces in the very viscous liquid orient all the particles perpendicular to the light beam, thus eliminating the possibility of any detectable amount of light being reflected in the direction of the camera.

The optical arrangements are identified in Fig. 6. Note that as thin a light beam as possible is desirable in order to reduce errors in the measured particle velocities. A beam having 
a width of $1 \mathrm{~mm}$ is found to be the lower practical limit with the equipment available. The primary purpose of the negatives obtained using diametral lighting is to determine particle velocities. However, when employing this side-lighting technique, the free surface of the liquid does not appear acceptably clear on the negative. A backlighting approach is therefore used when free surface profiles are desired. A floodlight is placed behind the glass tube, directly facing the camera, thus lighting the entite volume of the fluid rather than only a thin plane.

Figure 6 shows that the open-ended glass tube is surrounded by flat acrylic walls containing fluid of the same refractive index as that inside the tube. This is done to minimize refraction effects due to the curvature of the glass-liquid interface. Of course, when the free surface is photographed, the liquid surrounding the cylinder is removed. The effectiveness of these procedures is verified by measurements on photographs of a test grid and by calculations made using a full-scale drawing of the surfaces and liquids through which light rays pass after having been reflected by a glass sphere in the liquid. Errors in locating the position of a particle, due to refraction, are found to be well under 1 per cent.

Reflections from sources other than the tracer particles, particularly from the surface of the main glass tube, are an annoyance. They persist in spite of various precautions.

Measurements of particle velocities from consecutive negatives is accomplished using a comparator, i.e., a low-power microscope equipped with a movable table and having a reproducibility of about $10 \mu \mathrm{m}$. Thus, for a camera framing rate of $10 \mathrm{frames} / \mathrm{sec}$ and magnification of 0.2 , if the particle travels at $5 \mathrm{~cm} / \mathrm{sec}$, an error in measured velocity as low as 1 per cent could be obtained.

The experimental data are obtained primarily for average inlet velocities $\bar{V}$ of $2-7 \mathrm{~cm} / \mathrm{sec}$. The desirability of this range is established as follows. First, in order to eliminate refraction errors, the liquid must reach the wall of the main glass tube before any particle velocity data can be taken. For slow flows, a considerable time elapses until this occurs. This dictates that the digital computer program simulating the flow must run a relatively long time before results which can be compared with the experiment are generated. Computer economics therefore place one lower limit on desirable inlet velocities. Another independent lower limit is set by the accuracy to which small velocities can be measured. This in turn is determined by comparator accuracy, camera magnification (in turn a function of the field of view required) and the degree of nonlinearity of the particle acceleration.

The upper bound on average inlet velocity is determined by one of the two following criteria. Inlet velocities large enough to produce a jet prevent measurement of point velocities because in this case the liquid never fills the cylinder and refraction errors cannot be eliminated. Also, at high velocities, higher framing rates are required to prevent the images of the particles from streaking excessively. At these higher framing rates, the intensity of available illumination becomes a limiting factor.

Following the above guidelines, it is estimated, based on a cumulative error analysis, that for inlet velocities of $2-7 \mathrm{~cm} / \mathrm{sec}$, measured particle velocities are in error by less than 5 per cent. Note, therefore, that it is not possible to measure particle velocities accurately at every location within the liquid because of the wide range of velocities which exist.

A very accurate technique has been devised to determine the average inlet velocity for each individual run, since this is required as input data for the computer program. The method avoids direct consideration of viscosity variations from run to run, valve settings, pressure drop, or similar factors which would be involved in setting up a general calibration technique. Instead, use is made of the negatives themselves, and thus represents, in effect, a 
new calibration for each specific experiment. A negative well past $t=0$ is chosen at which time the cylindrical tube is nearly full, thus minimizing measuring errors. The volume of fluid in the cylinder is deduced from the photographic negative and is divided by the injection time and inlet area, thus yielding $\overline{\boldsymbol{V}}$.

A more detailed description of the various procedures and the experiment and equipment design is provided by Smith[19].

\section{EXPERIMENTAL AND NUMERICAL RESULTS}

The primary objective of the experimental program is to insure that the numerically calculated results conform reasonably with the actual physical situation, i.e., that no important phenomena have been neglected in the mathematical model and that the computer program is free from errors and accurate. Toward this end, a representative sampling of the total spectrum of results will be presented.

The finite-difference computations are performed on an IBM $360 / 67$ duplex multiprogramming system. The majority of the calculations involve either a $14 \times 14$ or $14 \times 28$ grid and cell dimensions of $\Delta r=\Delta z$. For a $14 \times 14$ grid, with a complete printing of results at a given time, the IBM 360 time consumed per time step ranges from 1 to $2 \mathrm{sec}$. The large amount of bookkeeping needed to keep track of the free surface undoubtedly accounts for a significant portion of this time. The calculations are generally continued for approximately 200 time steps. The computer program consists of 1200 FORTRAN statements.

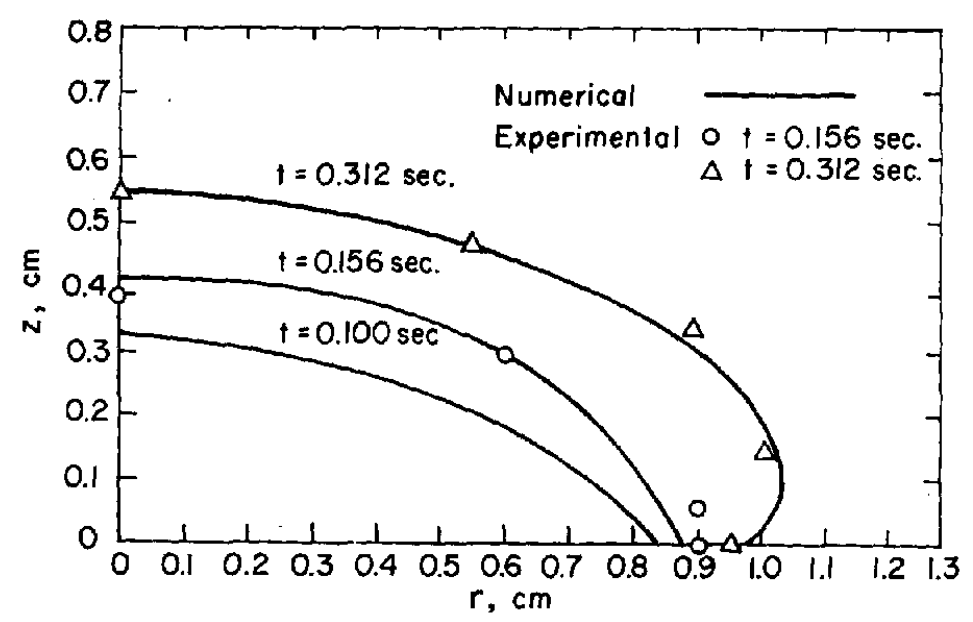

Fig. 7. Experimental and numerical surface profiles $(\mu=10$ poise, $\bar{V}=2.06 \mathrm{~cm} / \mathrm{sec}, \lambda=2.4$ ).

To convey an idea of the manner in which the fluid flows from the inlet tube into the vertical open-ended main cylindrical tube, three numerically calculated surface profiles are plotted in Fig. 7 for a liquid having a viscosity of 10 poise. For the times $t=0.156$ and $0.312 \mathrm{sec}$ the experimental data are also plotted. For this flow, $\operatorname{Re}=0.3$ and $\mathrm{Fr}=0.003$.

A further picture of the flow patterns for this run can be gained by referring to Fig. 8 . Here, the locations of the marker particles are displayed for $t=0.312 \mathrm{sec}$. Close examination reveals that at the interface between the empty cells and the cells which contain particles, the shape of the free surface is outlined (this is the basis for the curves in Fig. 7). The 


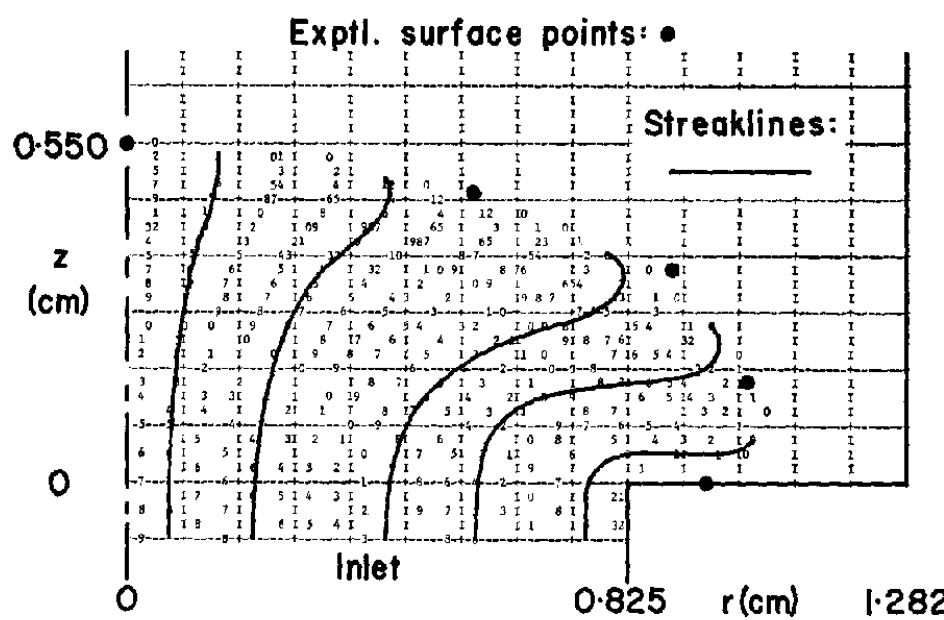

Fig. 8. Marker particle locations ( $\mu=10$ poise, $D=2.06 \mathrm{~cm} / \mathrm{sec}, \lambda=2.4$ ) at $t=0.312 \mathrm{sec}$, showing experimentally determined surface points $(O)$.

experimentally determined points which are plotted for $t=0.312 \mathrm{sec}$ on Fig. 7 are again shown on Fig. 8. The agreement is generally good. Streaklines are also shown in Fig. 8. These are drawn by connecting all marker particles entering the tube at the same radius. Note that the curvature of the leading edge of the fluid becomes appreciable as it progresses radially toward the wall. The neglect in the mathematical model of surface pressure caused by either surface tension or normal viscous stress may be responsible for the small difference between experiment and numerical computation in this region. A method of accounting for surface tension effects in free-surface flow has been published by Daly[4]. For flows which require more precise application of the free surface boundary conditions, various techniques are available (see, e.g., Hirt and Shannon[10], Nichols and Hirt[14]).

A time-step of $1 / 500$ of a second is used in these numerical calculations, resulting in a value of $\lambda$ of $2 \cdot 4$. Here,

$$
\lambda=\left[\frac{v}{(\Delta r)^{2}}+\frac{\left|v_{\max }\right|}{\Delta r}\right] \Delta t
$$

is a parameter that can be used to gauge the relative amount of viscous and convective momentum diffusion per time step, with some upper limit usually necessary for numerical stability. For example, with the ordinary explicit technique $\lambda$ would have to be less than about $0 \cdot 1$. Notice that the Dufort-Frankel formulation allows the use of a time step 24 times larger than this with a corresponding savings in computer time and cost.

The fluid configuration at a relatively advanced time in the flow of a 1-poise viscosity fluid is shown in Fig. 9. For this particular run, which uses a time-step of $1 / 350 \mathrm{sec}, \operatorname{Re}=12 \cdot 3$ and $\mathrm{Fr}=0 \cdot 4$. A picture of how the fluid deforms as it moves within the cavity or mold is obtained by connecting adjacent inlet particles and observing the subsequent distortion of this arrangement.

The velocity field corresponding to Fig. 9 is displayed in Fig. 10. Two experimentally determined tracer-particle velocities for this particular time are available for comparison as listed below. Their location is indicated by the two arrows on Fig. 10. 


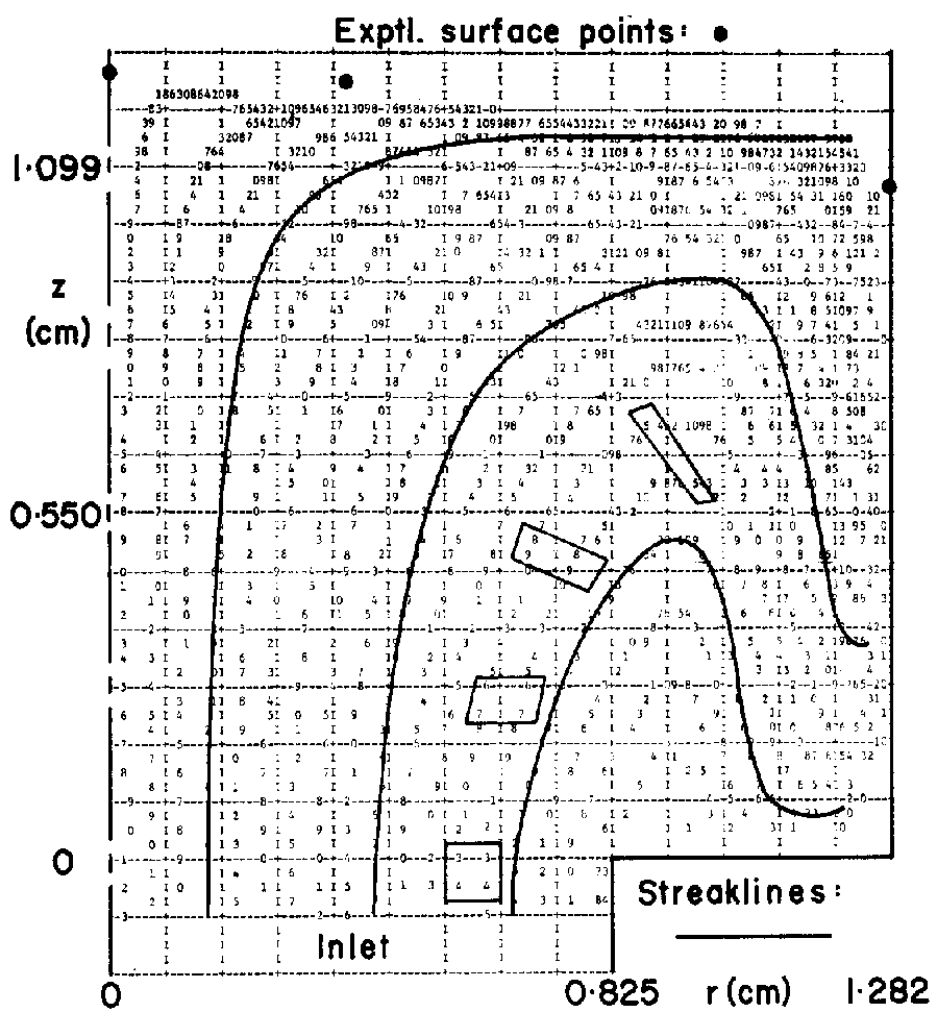

Fig. 9. Marker particle locations $(\mu=1$ poise, $\vec{\nabla}=7.37 \mathrm{~cm} / \mathrm{sec}, \lambda=0.6)$ at $t=0.38 \mathrm{sec}$, showing experimentally determined surface points $(\bigcirc)$.

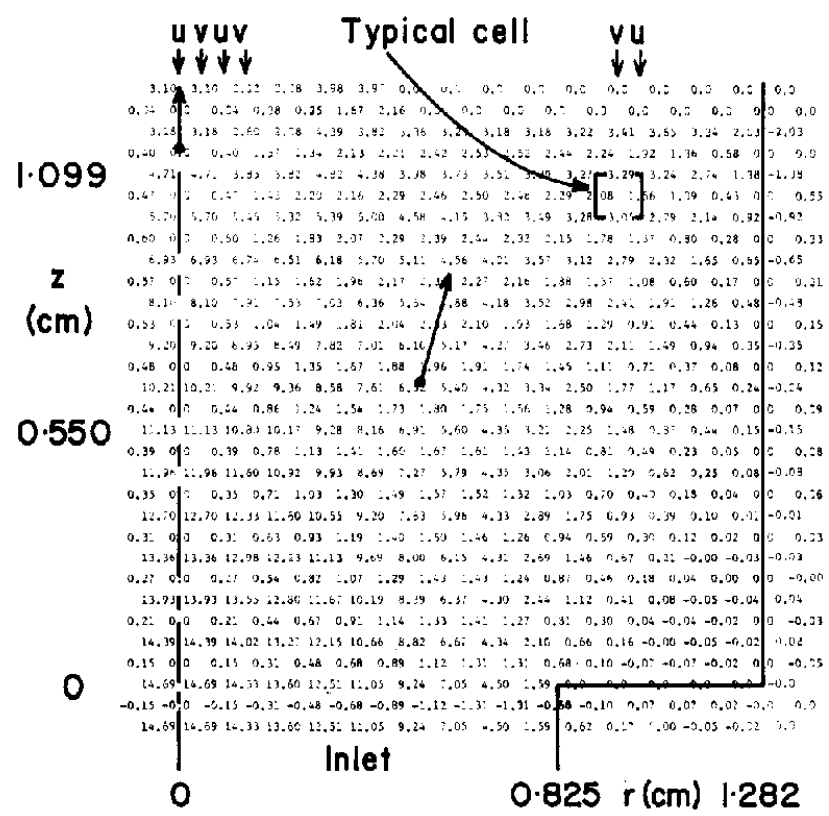

Fig. 10. Velocity field, $u$ and $v$ at $t=0.38 \mathrm{sec}(\mu=1$ poise, $\bar{V}=7.37 \mathrm{~cm} / \mathrm{sec}, \lambda=0.6)$. 


\begin{tabular}{cccccc}
\hline & & \multicolumn{3}{c}{ Experimental } & \multicolumn{2}{c}{ Numerical } \\
$r, \mathrm{~cm}$ & $z, \mathrm{~cm}$ & & $u$ & $v$ & \multicolumn{2}{c}{$u$} & $v$ \\
\hline 0.51 & 0.65 & 1.71 & 6.75 & 1.92 & 6.36 \\
0.00 & 1.15 & 0.00 & 3.54 & 0.00 & 3.86 \\
\hline
\end{tabular}

The magnitude of the experimentally determined vertical velocity component falls near the desired $5 \mathrm{~cm} / \mathrm{sec}$ needed to obtain good measuring accuracy. The experimental and numerical values of $v$ are seen to agree within 5-10 per cent (obviously, it is possible for both the experimental and numerical values to be within 5 per cent of the true velocity and show a relative error of up to 10 per cent). Correspondingly poorer agreement is obtained for the lower velocities. Considering the overall complexity of the problem, the observed agreement, which is typical of that obtained for other runs, can be considered good and serves, together with the surface profile data, to validate the computational technique.

Note in Fig. 10 that the region near the corner is bypassed and that a large vortex is developing. This is in agreement with the computations of Macagno and Hung[12]. To provide a further idea of the behavior of the velocity field, some radial profiles for $u$ and $v$ are taken from Fig. 10 and plotted on Fig. 11. The pressure field corresponding to Fig. 10 is given in Fig. 12. Recall that the pressure in the surface and empty cells is taken as zero.

Proceeding to a run having a considerably higher inlet velocity, Fig. 13 consists of few typical photographs used to measure surface profiles. The surface profiles for the computer simulation of this run (not shown) are considerably advanced relative to the experimental profiles, indicating that the assumption in the mathematical model of the existence of a known parabolic profile at $t=0$ is introducing a larger error at this lower viscosity and higher inlet velocity. For this type of situation it may be better to start the numerical calculations in the inlet tube.

A computer simulation has also been performed for the above flow rate but with a viscosity of 60 poise, thus yielding $\lambda=13 \cdot 0$. No numerical instability occurred.

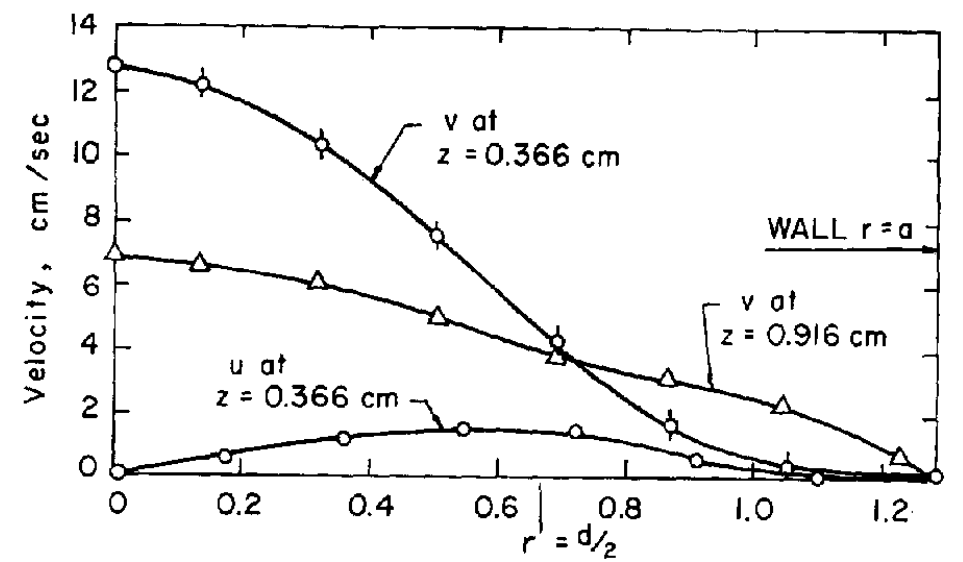

Fig. 11. Radial velocity profiles for $u$ and $v$ at $t=0.38 \mathrm{sec}(\mu=1$ poise, $\bar{V}=7.37 \mathrm{~cm} / \mathrm{sec}$. $\lambda=0.6$ ). 


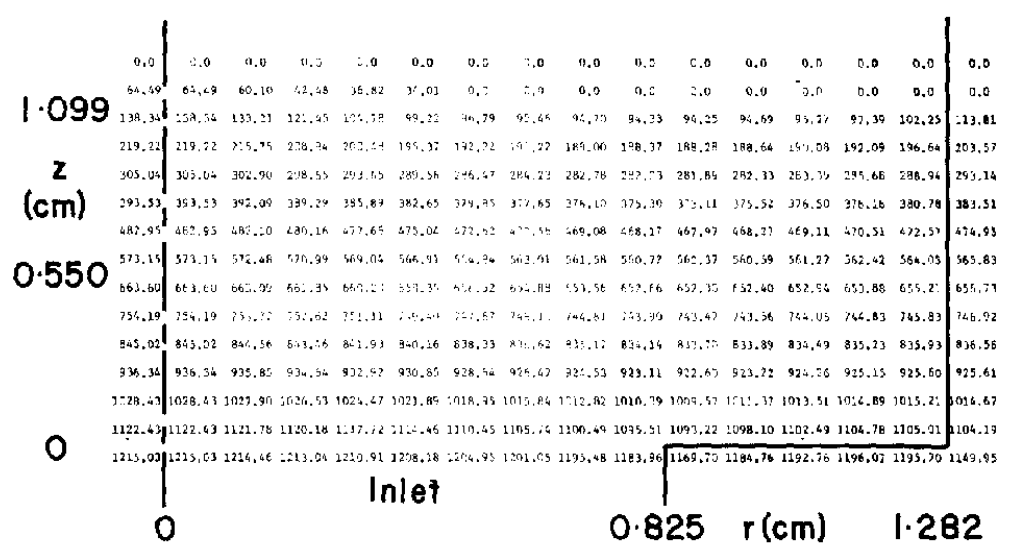

Fig. 12. Pressure field at $t=0.38 \mathrm{sec}(\mu=1$ poise, $\bar{V}=7.37 \mathrm{~cm} / \mathrm{sec}, \lambda=0.6)$; units of pressure for $\phi$ are $\mathrm{cm}^{2} / \mathrm{sec}^{2}$.

An additional verification of the computer program can be deduced by determining if it correctly predicts the conditions for the formation of a jet. Quite obviously, the definition of an unsteady jet in a gravitational field is somewhat arbitrary. Assume for the sake of argument that a jet occurs if the fluid loses, due to gravitational force, less than 15 per cent of its original momentum while traversing a vertical distance of two $\mathrm{cm}$. Under these conditions, the diameter will remain approximately that of the inlet diameter. It can easily be shown, using a momentum balance, that a jet will then form for an average inlet velocity

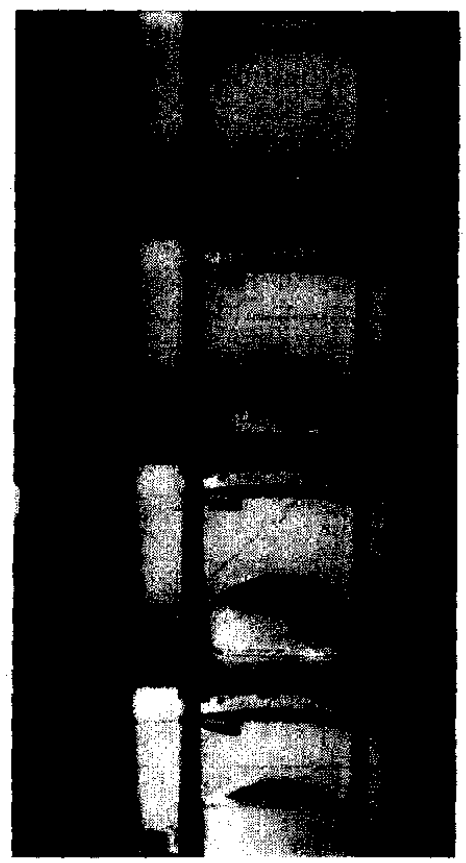

Fig. 13. Photographs ( $\mu=1$ poise, $\vec{V}=23 \cdot 2 \mathrm{~cm} / \mathrm{sec}$ ). 
of approximately $100 \mathrm{~cm} / \mathrm{sec}$ regardless, of course, of the viscosity. For a tube diameter of $1.67 \mathrm{~cm}$, this indicates that $F_{r_{j e t}}=5$. Both the computer runs and experiments agree with this criterion. Various other checks that verify the correctness of the numerical results have also been made.

To further consolidate the concepts discussed above, Fig. 14 represents a series of com-

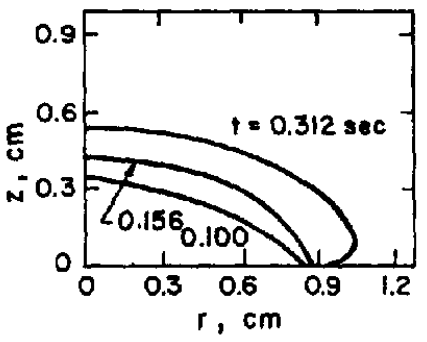

(a)

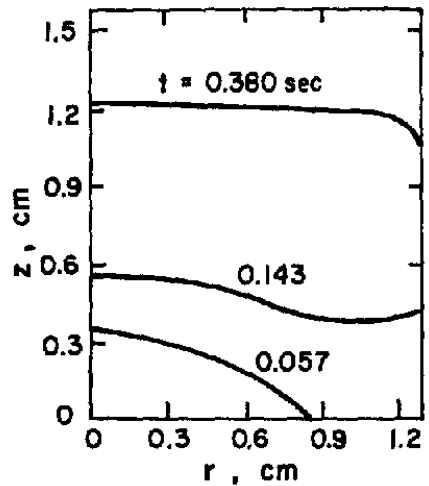

(c)

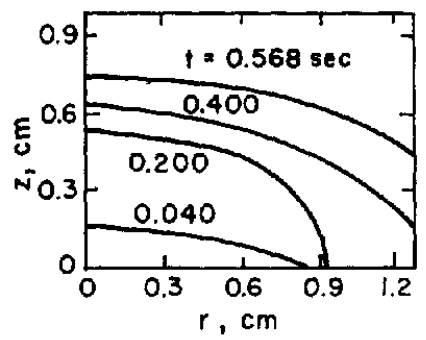

(b)

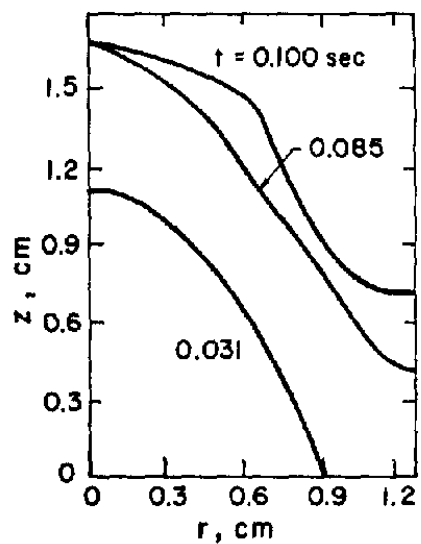

(d)

Fig. 14. Time dependent surface profiles: (a) $\mu=10$ poise, $\bar{V}=2.06 \mathrm{~cm} / \mathrm{sec}$ (b) $\mu=10$ poise, $\bar{V}=2.58 \mathrm{~cm} / \mathrm{sec}$ (c) $\mu=1$ poise, $\bar{V}=7.37 \mathrm{~cm} / \mathrm{sec}$ (d) $\mu=1$ poise, $\bar{V}=23.2 \mathrm{~cm} / \mathrm{sec}$.

puter generated surface profiles, in the order of increasing average inlet velocity. This type of presentation, although interesting, suffers the disadvantage of lacking streakline information. Accordingly, it is difficult to visualize accurately the general movement of various fluid segments over a given time span. The marker particles, as used in previous figures, alleviated this difficulty.

Finally, it is of interest to summarize certain observed stability characteristics of the numerical solution. First and most important, the strong partial instability of the diffusion terms has been inhibited, as evidenced above. This permits the study of highly viscous fluids using sensible values for the time step. Second, the computations usually become unstable after approximately 200 time steps. The lack of long-term integrability is most likely to be due to the weak partial instability alluded to previously as 'time splitting.' This contention is based on the observation that instability occurs independently of the smallness of the time step. A periodic averaging procedure would no doubt have suppressed its appearance (Williams[23]). 


\section{REFERENCES}

1. Andrade E. N. daC. and Tsien L. C., The velocity distribution in a liquid-into-liquid jet, Proc. Phys. Soc. 49, 381 (1937).

2. Atkinson B, Kemblowski Z. and Smith J. M., Measurements of velocity profile in developing liquid flows, A.I.Ch.E.J. 13, 17 (1967).

3. Carnahan B., Luther H. A. and Wilkes J. O., Applied Numerical Methods. Wiley, New York (1969).

4. Daly B. J., A technique for including surface tension effects in hydrodynamic calculations, J. Comp. Phys. 4, 97 (1969).

5. Dufort E. C. and Frankel S. P., Stability conditions in the numerical treatment of parabolic differential equations, Mathematical Tables and Other Aids to Computation 7, 135 (1953).

6. Fox L., Numerical Solution of Ordinary and Partial Differential Equations. Pergamon Press, Oxford (1962).

7. Gerrard, J. E., Steidler F. E. and Appeldoorn J. K., Viscous heating in capillaries. The isothermal-wall case, Ind. Eng. Chem. Fund. 5, 260 (1966).

8. Goren S. L. and Wronski S., The shape of low-speed capillary jets of Newtonian liquids, J. Fluid Mech. $25,185(1966)$

9. Harlow F. H. and Welch J. E., Numerical study of large-amplitude free-surface motions, Phys. Fluids, 9,842 (1966).

10. Hirt C. W. and Shannon J. P., Free-surface stress conditions for incompressible-flow calculations, J. Comp. Phys. 2, 403 (1968).

11. Huh C. and Scriven L. E., Hydrodynamic model of steady movement of a solid/liquid/fluid contact line, J. Coll. Interface Sci. 35, 85 (1971).

12. Macagno E. O. and Hung T. K. Computational and experimental study of a captive annular eddy, J. Fluid Mech. 28, 43 (1967).

13. Middleman S. and Gavis J., Expansion and contraction of capillary jets of Newtonian liquids, Phys. Fluids 4, 355 (1961).

14. Nichols B. D. and Hirt C. W., Improved free-surface boundary conditions for numerical incompressibleflow calculations, $J$. Comp. Phis. 8, 434 (1971).

15. Feebles F. N. and Liu K. C., Photoviscous analysis of two-dimensional laminar flow in an expanding jet, Experimental Mech. 5, 229 (1965).

16. Platzman G. N., The lattice structure of the finite difference primitive and vorticity equations, Mon. Weath. Rev. 86, 285 (1958).

17. Richardson S., A 'stick-slip' problem related to the motion of a free jet at low Reynolds numbers, Proc. Camb. Phil. Sor. 67, 477 (1970).

18. Schlichting H., Boundary Layer Theory. McGraw-Hill, New York (1959).

19. Smith T. G., An investigation of free-surface flow into a vertical cylindrical enclosure, Ph.D. Dissertation, The University of Michigan (1970).

20. Talbot L., Laminar swirling pipe flow, J. Appl. Mech. 21, 1 (1954).

21. Tillett J. P. K., On the laminar flow in a free jet of liquid at high Reynolds numbers, J. Fluid Mech. 32, $273(1968)$.

22. Welch J. E., Harlow, F. H., Shannon J. P. and Daly B. J., The MAC method-a computing technique for solving viscous incompressible, transient fluid-flow problems involving free-surfaces, Los Alamos Sci, Lab. LA-3425, 1965

23. Williams G.P., Numerical integration of the three-dimensional Navier-Stokes equations for incompressible flow, J. Fluid Mech. 37, 727 (1969). 